\title{
Validation of Human Three-Joint Arm's Optimal Control Model with Hand-Joint's Feedback Mechanism Based on Reproducing Constrained Reaching Movements*
}

\author{
Toshikazu MATSUI** and Nobuaki NAKAZAWA** \\ **Graduate School of Engineering, Gunma University \\ 29-1 Honcho, Ota-shi, Gunma 373-0057, Japan \\ E-mail:matsui@gunma-u.ac.jp, n.nakazawa@gunma-u.ac.jp
}

\begin{abstract}
We have already formulated the human three-joint arm's optimal control model with its hand-joint's feedback mechanism and have clarified that the model is effective in reproducing human unconstrained reaching movements including the experimental fact that the hand-joint angle hardly changes over the entire movement. For validating the proposed model, this paper applies it to reproducing human constrained reaching movements in which the hand has to take a specified posture at the final point as in the case of holding a cup's handle with fingertips. The following results were obtained: (1) the proposed model was able to move its hand joint despite the existence of the feedback mechanism preventing the hand-joint angle from changing; (2) the proposed model succeeded at reproducing the measured hand trajectories and the measured angular transition characteristics for the hand joint in most of the constrained reaching movements. These results suggest that the proposed model has the ability to simulate human reaching movements regardless of whether they are unconstrained or constrained, i.e., it can be a general and plausible model for the human three-joint arm's control mechanism. Moreover, it is computationally considered that the hand-joint's angular transition characteristic depends on the average of the resultant torque around the hand joint over the entire movement.
\end{abstract}

Key words: Three-Joint Arm, Optimal Control Model, Hand Joint, Feedback Torque, Constrained Movement, Hand Posture, Unconstrained Movement, Angular Transition, Resultant Torque

\section{Introduction}

So far, three typical kinds of optimal control models have been proposed to reproduce human arm's two-point reaching movements: minimum jerk model ${ }^{(1)}$, minimum torquechange $\operatorname{model}^{(2)(3)}$, and minimum energy model ${ }^{(4)(5)}$. They predict the optimal hand trajectory by minimizing the square of jerk of the hand position, the square of the rate of change of torque, and the consumed energy, respectively, integrated over the entire movement. Although these models can reproduce fundamental characteristics for human arm's reaching movements, they have no ability to reproduce the variability of end points of the hand paths because their end-point boundary conditions are strictly determined. As models to reproduce the end-point variability as well as reaching movement characteristics, two kinds of models have been proposed. One is the minimum variance model that 
determines the optimal hand trajectory by minimizing the variance in the fluctuation of the end points over a given period after the reaching movement ${ }^{(6)}$. The other is the modified minimum torque-change model formulated by modifying the end-point boundary conditions of the minimum torque-change model from fixed ones into free ones ${ }^{(7)}$. All the above models are two-joint models composed of shoulder and elbow joints. However, in order to accurately simulate human arm movements including grasping ones, it is desirable that the human arm's control mechanism should be formulated at least as a three-joint model including a hand joint. We have already formulated such a three-joint arm's optimal control model characterized by a feedback mechanism in its hand joint and energy minimization, and have clarified that this model can reproduce not only human unconstrained reaching movements in which the arm is moved with no constraint but also the experimental fact that as for unconstrained reaching movements, the hand-joint angle hardly changes over the entire movement ${ }^{(8)}$ : that is, the hand-joint's feedback mechanism has the function to prevent the hand joint from moving. However, it is not verified whether the model can be applied to reproducing human constrained reaching movements in which the hand joint is required to move during the movement, in other words, whether the model can move its hand joint in spite of the existence of the hand-joint's feedback mechanism preventing its hand-joint angle from changing. If the proposed model can be a general and plausible model for the human three-joint arm's control mechanism, it should be able to move its hand joint for such constrained reaching movements. Therefore, the verification is very significant for clarifying the proposed model's effectiveness.

From the above point of view, this paper applies the proposed three-joint arm model to reproducing human three-joint arm's constrained reaching movements accompanied by evident motion in the hand joint, and clarifies the model's effectiveness. Actually, we compare the hand trajectories predicted optimally by the proposed model with those measured experimentally for such constrained reaching movements, and elucidate the model's reproducible performance. The constrained reaching movements used in the experiment were movements in which the hand had to take a specified posture at each final target position; the hand posture was realized by moving the hand joint during the movement so that the end of the hand pointed in the direction of 90 or 180 degrees from the $\mathrm{X}$-axis at the final point. That is, the constrained reaching movements were assumed to be reaching movements for holding the handle of a cup with fingertips. Consequently, the following results were obtained: (1) the proposed model was able to move its hand joint despite the existence of the hand-joint's feedback mechanism; (2) the hand trajectories predicted by the model and the hand-joint angular transition characteristics, i.e., hand-joint angle profiles, predicted by the model agreed well with those measured experimentally for most of the human arm's constrained reaching movements used in the experiment. These results suggest that the proposed model has the ability to simulate human three-joint arm's constrained reaching movements accompanied by evident motion in the hand joint as well as unconstrained ones accompanied by little motion, in other words, it can be effective as a general and plausible model for the human three-joint arm's control mechanism. Section 2 briefly explains the proposed three-joint arm's optimal control model. In Section 3, we measure human three-joint arm's constrained reaching movements in which the hand has to take a specified posture at each target position, and clarify the model's performance in reproducing the measured hand trajectories and the measured hand-joint's angular transition characteristics. In Section 4, we consider computationally how the hand-joint's feedback mechanism functions to reproduce both unconstrained and constrained reaching movements.

\section{Three-Joint Arm's Optimal Control Model}

It was experimentally clarified that for human three-joint arm's unconstrained reaching 
movements in which the arm is moved with no constraint, the human hand-joint angle hardly changes over the entire movement ${ }^{(8)}$. This experimental fact is apparently similar to the freezing phenomenon ${ }^{(9) \sim(11)}$ in which the stiffness of joints is strengthened by contracting agonist and antagonist muscles together. In terms of the outward similarity, we regarded the experimental fact as a freezing-like phenomenon and formulated its mechanism as a feedback controller that produced the feedback torque in the direction opposite to the hand-joint motion $^{(8)}$. Letting a desired hand-joint angle, its angular velocity, and its angular acceleration be all zero in the feedback controller, the feedback torque $\tau_{3}{ }^{*}$ around the hand joint can be expressed as follows.

$$
\tau_{3}^{*}=-k_{a} \ddot{\theta}_{3}-k_{v} \dot{\theta}_{3}-k_{p} \theta_{3}
$$

where $k a, k v$, and $k p$ are the feedback gains. Actually, according to our pre-calculation, the feedback gain $k a$ was set to 0.147 (300 times as large as the moment of inertia of the hand), and the other feedback gains $k v$ and $k p$ were set to zero ${ }^{(8)}$. That is because, when the feedback gains $k v$ and $k p$ were not null, the feedback torque $\tau_{3}{ }^{*}$ made the model's hand joint oscillate. Therefore, assuming that the human three-joint arm model illustrated in Figure 1 has the freezing-like mechanism in its hand joint, its equation of motion can be given as follows by adding the feedback torque $\tau_{3}^{*}$ to the hand-joint torque $\tau_{3}$ :

$$
\begin{aligned}
& \quad \tau *=\boldsymbol{M} \ddot{\boldsymbol{\theta}}+\boldsymbol{h}+\boldsymbol{D} \dot{\boldsymbol{\theta}} \\
& \tau^{*}=\left[\tau_{1}, \tau_{2}, \tau_{3}+\tau_{3}\right]^{\mathrm{T}}, \quad \boldsymbol{\theta}=\left[\theta_{1}, \theta_{2}, \theta_{3}\right]^{\mathrm{T}} \\
& M_{11}=J_{1}+J_{2}+J_{3}+2 b_{12} \cos \left(\theta_{2}\right)+2 b_{13} \cos \left(\theta_{2}+\theta_{3}\right)+2 b_{23} \cos \left(\theta_{3}\right) \\
& M_{12}=M_{21}=J_{2}+J_{3}+b_{12} \cos \left(\theta_{2}\right)+b_{13} \cos \left(\theta_{2}+\theta_{3}\right)+2 b_{23} \cos \left(\theta_{3}\right) \\
& M_{13}=M_{31}=J_{3}+b_{13} \cos \left(\theta_{2}+\theta_{3}\right)+b_{23} \cos \left(\theta_{3}\right), M_{33}=J_{3} \\
& M_{22}=J_{2}+J_{3}+2 b_{23} \cos \left(\theta_{3}\right), M_{23}=M_{32}=J_{3}+b_{23} \cos \left(\theta_{3}\right) \\
& J_{1}=m_{1} L_{c 1}{ }^{2}+\left(m_{2}+m_{3}+m_{L}\right) L_{1}^{2}+I_{1} \\
& J_{2}=m_{2} L_{c 2}{ }^{2}+\left(m_{3}+m_{L}\right) L_{2}^{2}+I_{2}, J_{3}=m_{3} L_{c 3}{ }^{2}+m_{L} L_{3}{ }^{2}+I_{3} \\
& b_{12}=m_{2} L_{1} L_{c 2}+\left(m_{3}+m_{L}\right) L_{1} L_{2}, b_{13}=m_{3} L_{1} L_{c 3}+m_{L} L_{1} L_{3}, b_{23}=m_{3} L_{2} L_{c 3}+m_{L} L_{2} L_{3} \\
& h_{1}=\left\{-b_{12} \sin \left(\theta_{2}\right)-b_{13} \sin \left(\theta_{2}+\theta_{3}\right)\right\}\left(\dot{\theta}_{2}{ }^{2}+2 \dot{\theta}_{1} \dot{\theta}_{2}\right)-\left\{b_{13} \sin \left(\theta_{2}+\theta_{3}\right)+b_{23} \sin \left(\theta_{3}\right)\right\}\left(\dot{\theta}_{3}{ }^{2}+2 \dot{\theta}_{2} \dot{\theta}_{3}+2 \dot{\theta}_{1} \dot{\theta}_{3}\right) \\
& h_{2}=\left\{b_{12} \sin \left(\theta_{2}\right)+b_{13} \sin \left(\theta_{2}+\theta_{3}\right)\right\} \dot{\theta}_{1}^{2}-b_{23} \sin \left(\theta_{3}\right)\left(\dot{\theta}_{3}^{2}+2 \dot{\theta}_{2} \dot{\theta}_{3}+2 \dot{\theta}_{1} \dot{\theta}_{3}\right) \\
& h_{3}=\left\{b_{13} \sin \left(\theta_{2}+\theta_{3}\right)+b_{23} \sin \left(\theta_{3}\right)\right\} \dot{\theta}_{1}^{2}+b_{23} \sin \left(\theta_{3}\right)\left(\dot{\theta}_{2}{ }^{2}+2 \dot{\theta}_{1} \dot{\theta}_{2}\right)
\end{aligned}
$$

where $\theta_{i}, \dot{\theta}_{i}, \ddot{\theta}_{i}$, and $\tau_{i}$ denote the angle, the angular velocity, the angular acceleration, and the drive torque of the $i$-th joint, respectively. Links 1, 2, and 3 correspond to the upper arm, the forearm, and the hand, respectively, and joints 1, 2, and 3 correspond to the shoulder, elbow, and hand joints, respectively. The shoulder is the origin of the X-Y coordinates. $\boldsymbol{M}=[\mathrm{Mij}]$ $(i, j=1 \sim 3), \boldsymbol{h}=\left[h_{1}, h_{2}, h_{3}\right]^{\mathrm{T}}$, and $\boldsymbol{D}$ represent the inertia matrix, the Coriolis and centrifugal

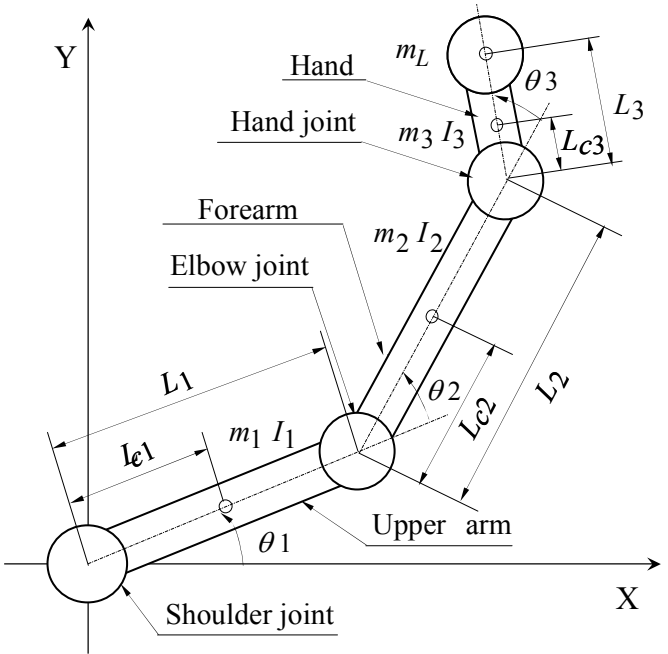

Table 1 Dynamics parameters of the human three-joint arm for one subject

Fig.1 A model of the human three-joint arm.

\begin{tabular}{|c|c|c|c|}
\hline parameter & Link1 & Link2 & Link3 \\
\hline$L i[\mathrm{~m}]$ & 0.276 & 0.257 & 0.130 \\
\hline$L c i[\mathrm{~m}]$ & 0.141 & 0.086 & 0.043 \\
\hline$m i[\mathrm{~kg}]$ & 1.784 & 0.951 & 0.481 \\
\hline$I i\left[\mathrm{~kg} \cdot \mathrm{m}^{2}\right]$ & 0.0127 & 0.0041 & 0.0005 \\
\hline$D i[\mathrm{Nm} \cdot \mathrm{s} / \mathrm{rad}]$ & 0.370 & 0.290 & 0.190 \\
\hline
\end{tabular}


force vector, and the $3 \times 3$ diagonal viscous matrix, respectively; superscript $\mathrm{T}$ denotes the transpose of a vector; $m_{i}$ and $m_{L}$ represent the mass of the $i$-th link and the mass $(=1.2 \mathrm{~kg}) \mathrm{of}$ the object (handle) held with the fingertips, respectively; $L i, L c i$, and $I i$ are the length of the $i$-th link, the length from the center of mass of the $i$-th link to the $i$-th joint, and the moment of inertia around the center of mass of the $i$-th link, respectively; $D i$ is the viscosity coefficient of the $i$-th joint. Since the human arm model shown in Fig. 1 moves within a horizontal plane at the height of the shoulder, the influence of gravity can be neglected. Table 1 summarizes the physical parameters of the arm for one subject. The values of $m_{i}$, $L c i$, $I i$, and $D i(i=1 \sim 3)$ were estimated from the measured link length $L i$ for each subject by referring to references (3) and (5). Substituting Eq. (1) into Eq. (2), the following state equation can be given:

$$
\begin{aligned}
& \dot{\boldsymbol{x}}=\left[\begin{array}{c}
\dot{\boldsymbol{\theta}} \\
-\underline{\boldsymbol{M}}^{-1}(\boldsymbol{h}+\boldsymbol{D} \dot{\boldsymbol{\theta}})+\underline{\boldsymbol{M}}^{-1} \tau \\
\boldsymbol{0}_{(3 \times 1)}
\end{array}\right]+\left[\begin{array}{c}
\boldsymbol{0}_{(3 \times 3)} \\
\boldsymbol{0}_{(3 \times 3)} \\
\boldsymbol{I}
\end{array}\right] \dot{\tau}=A(\boldsymbol{x})+B \boldsymbol{u} \\
& \boldsymbol{x}=\left[\theta_{1}, \theta_{2}, \theta_{3}, \dot{\theta}_{1}, \dot{\theta}_{2}, \dot{\theta}_{3}, \tau_{1}, \tau_{2}, \tau_{3}\right]^{\mathrm{T}}, \boldsymbol{u}=\dot{\boldsymbol{\tau}}=\left[\dot{\tau}_{1}, \dot{\tau}_{2}, \dot{\tau}_{3}\right]^{\mathrm{T}}, \boldsymbol{I}: \text { unit matrix }(3 \times 3)
\end{aligned}
$$

where $\boldsymbol{x}$ and $\boldsymbol{u}$ represent the state variable vector and the input variable vector, i.e., the rate of change of torque, respectively; $\underline{\boldsymbol{M}}$ is the matrix identical to the inertia matrix $\boldsymbol{M}$ except that the $(3,3)$ element of $\boldsymbol{M}$ is replaced by $M_{33}+k a$. Therefore, incorporating the feedback mechanism into the model's hand joint is equivalent to increasing the $(3,3)$ element of $\boldsymbol{M}$ to make the hand joint difficult to move. The subscripts $(3 \times 1)$ and $(3 \times 3)$ in Eq. (3) represent the element size of the zero vector and the zero matrix, respectively.

The proposed optimal control model predicts the optimal hand trajectory by minimizing the following criterion function subject to the constraint imposed by the state equation of Eq. (3).

$$
\begin{aligned}
J & =\left\{\boldsymbol{x}\left(t_{f}\right)-\boldsymbol{x}_{t f}\right\}^{\mathrm{T}} \boldsymbol{Q}\left\{\boldsymbol{x}\left(t_{f}\right)-\boldsymbol{x}_{t f}\right\}+\int_{0}^{t_{f}}\left(W+\omega_{4} \boldsymbol{u}^{\mathrm{T}} \boldsymbol{R} \boldsymbol{u}\right) d t \\
W & =\omega_{1} \tau^{\mathrm{T}} \dot{\boldsymbol{\theta}}+\omega_{2} \tau^{\mathrm{T}} \tau+\omega_{3} \dot{\boldsymbol{\theta}}^{\mathrm{T}} \boldsymbol{D} \dot{\boldsymbol{\theta}}
\end{aligned}
$$

The first term of the criterion function (Eq. (4)) represents the error between the state variable at the final point and its desired value, and the second term of $J$ represents the weighted sum of three kinds of energy costs and the square of the torque-change integrated over the entire movement. The first, second, and third costs of the energy term $W$ (Eq. (5)) represent the moment power of joints, the energy for sustaining the torque of joints, and the energy consumed by viscous resistance, respectively, and $\omega_{1}, \omega_{2}, \omega_{3}$, and $\omega_{4}$ are the weight values. In Eq. (4), $t_{f}$ is the movement duration; $\boldsymbol{x}\left(t_{f}\right)$ and $\boldsymbol{x}_{t f}$ are the value of the state variable $\boldsymbol{x}$ at the final point $\left(t=t_{f}\right)$ and its desired value, respectively; $\boldsymbol{Q}$ and $\boldsymbol{R}$ are the $9 \times 9$ and $3 \times 3$ diagonal weight matrices, respectively. It would also be possible to replace the scalar weights $\omega_{1}, \omega_{2}, \omega_{3}$, and $\omega_{4}$ with diagonal weight matrices like $\boldsymbol{Q}$ and $\boldsymbol{R}$. However, the scalar weights are used to make the model as simple as possible and are based on the idea that, if human arm's reaching movement characteristics can be reproduced by a simpler model, it should be more desirable. The optimization of Eq. (4) can be solved based on the calculus of variations. Letting a Hamiltonian be defined as

$$
H(\boldsymbol{x}, \boldsymbol{u}, \lambda)=W+\omega_{4} \boldsymbol{u}^{\mathrm{T}} \boldsymbol{R} \boldsymbol{u}+\lambda^{\mathrm{T}}(\boldsymbol{A}(\boldsymbol{x})+\boldsymbol{B} \boldsymbol{u}),
$$

the control which optimizes the criterion function of Eq. (4) can be derived from the following equation.

$$
\partial H / \partial \boldsymbol{u}=\boldsymbol{0} .
$$

The optimal hand trajectory can be determined by solving the following set of nonlinear differential equations with respect to $\boldsymbol{x}$ and $\lambda$, which is a necessary condition for an optimum point to exist, under the optimal control of Eq. (7).

$$
\boldsymbol{x}=\partial H / \partial \lambda, \quad \lambda=-\partial H / \partial \boldsymbol{x}
$$

where $\lambda$ denotes the adjoint variable vector with 9 components. The boundary conditions are as follows: 


$$
\boldsymbol{x}(0)=\boldsymbol{x}_{0}, \lambda\left(t_{f}\right)=2 \boldsymbol{Q}\left\{\boldsymbol{x}\left(t_{f}\right)-\boldsymbol{x}_{t f}\right\},
$$

where $\boldsymbol{x}_{0}$ and $\lambda\left(t_{f}\right)$ are the initial value of the state variable $\boldsymbol{x}$ at the start point $(t=0)$ and the final value of the adjoint variable $\lambda$ at the final point $\left(t=t_{f}\right)$, respectively. Therefore, Eq. (8) becomes a two-point boundary-value problem. This two-point boundary-value problem can be solved iteratively based on a Newton-like method.

\section{Model's Performance in Reproducing Constrained Reaching Movements}

\subsection{Measurement of Constrained Reaching Movements}

Figure 2 shows the apparatus for measuring horizontally planar reaching movements. In the experiment, the subjects seated and held a hand-held bar $\left(m_{L}=1.2 \mathrm{~kg}\right)$ fixed at the end of a two-link mechanical manipulator with their fingertips, and performed horizontal two-point reaching movements toward the green LED targets mounted in the desk covered with a transparent Plexiglas sheet at the shoulder level. Figure 3 shows the arrangement of the start positions (S1 and S2) and the target positions (T4, T5, T6, T7, T8). A red LED was mounted on each of the three joints and the hand-held bar. Hand paths from the start positions to the final positions were derived from recording the motion of the red LEDs with a digital video camera ( 30 frames/sec) placed above the experimental apparatus and analyzing it. The shoulder joint was set as the origin of the $\mathrm{X}-\mathrm{Y}$ coordinates.

The two-point reaching movements used in the experiment were constrained ones in which the hand joint was required to move during the movement. The subjects were instructed to move their arm so that their hand took a specified posture at the target point; the hand posture was realized by moving their hand joint during the movement so that the end of their hand pointed in the direction of 90 or 180 degrees from the X-axis at $t=t_{f}$. Moreover, they were instructed to do so toward the left or right direction: as for leftward movements, their hand was moved leftward from the start position S1 (the same position as T7) to one of the target positions T4, T5, and T6; as for rightward movements, their hand was moved rightward from the start position S2 (the same position as T5) to one of the target positions $\mathrm{T} 7$ and T8. Figure 4 shows the hand postures required at the target positions; (a) and (b) are for leftward movements to T4 or T6 and rightward movements to $\mathrm{T} 7$ or T8, respectively, and the end of the hand at $t=t_{f}$ points in the direction orthogonal to

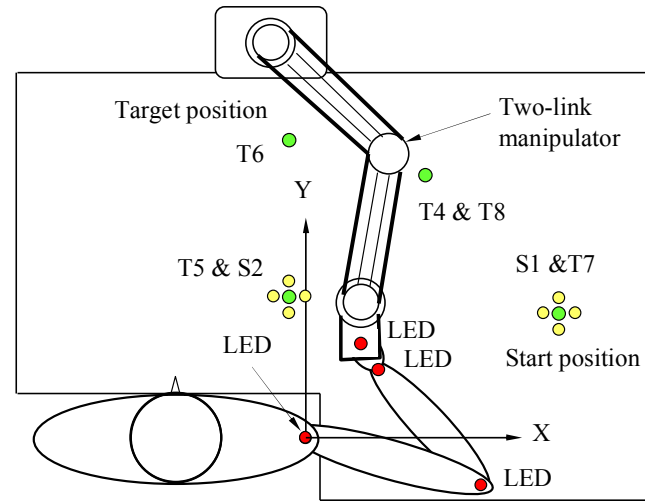

Fig.2 Experimental setup for measuring reaching movements

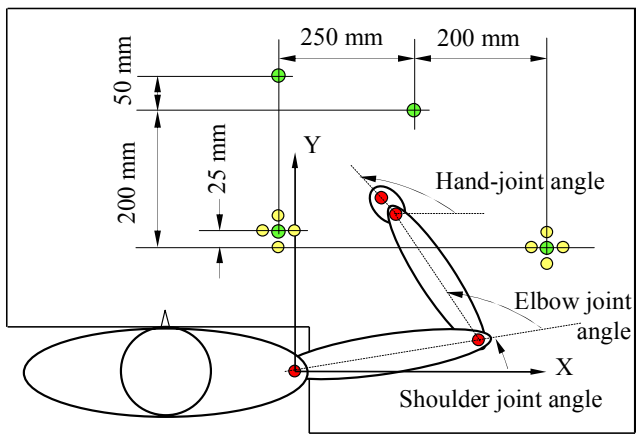

Fig.3 Arrangement of the start and target LEDs

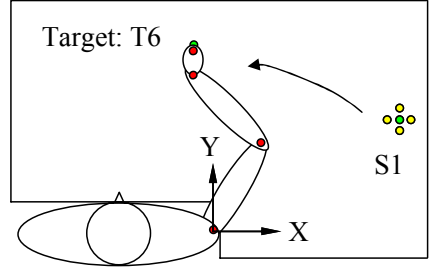

(a) Orthogonal condition (T4, T6)

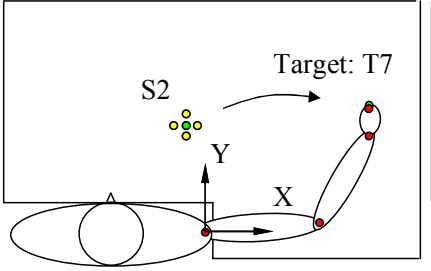

(b) Orthogonal condition (T7, T8)

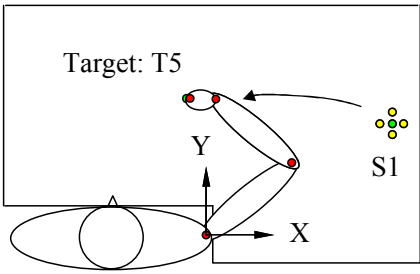

(c) Parallel condition (T5)

Fig.4 Hand postures required at the target positions for the constrained reaching movements 
the frontal parallel plane (orthogonal condition); (c) is for the leftward movement to T5, and the end of the hand at $t=t_{f}$ points in the direction parallel to the frontal parallel plane (parallel condition). The experiment was executed under the above conditions according to the following procedure.

(1) The subjects move the hand-held bar to the start LED (S1 or S2) while it is illuminated.

(2) All the target LEDs are illuminated for two seconds, and then they are turned off for a randomly changed duration between 1 and $2 \mathrm{sec}$.

(3) As soon as one randomly selected target LED is lit, the subjects move the hand-held bar from the start position to the target position under the orthogonal or parallel condition illustrated in Fig. 4.

These constrained reaching movements were assumed to be reaching movements for holding the handle of a cup with fingertips. That is, the orthogonal condition corresponded to the case where the handle was on the near side of a cup, whereas the parallel condition corresponded to the case where the handle was on the right hand side of a cup. Two subjects (T.S. and K.T.) participated in the experiment. Since 15 trials were performed for each target, a total of 45 trials and 30 trials were executed as the leftward and rightward movements, respectively. The experiment was conducted in a dark room. Off-line digital image signal processing was performed to compute the subjects' hand paths and hand-joint angles, and the shoulder- and elbow-joint angles were derived from the hand paths and the hand-joint angles based on the inverse kinematics. To multiply the number of measured data, the joint angles every $1 / 30 \mathrm{sec}$ were interpolated into those every $1 \mathrm{msec}$ using a leastsquared method with the condition that the joint angles, their angular velocities, and their angular accelerations agreed with the measured ones at the end positions, and then the interpolated joint angles were differentiated to calculate their angular velocities and differentiated again to calculate their angular accelerations.

Figure 5 shows the hand paths derived from the constrained reaching movements measured experimentally; (a) is for the leftward movements from S1 to T4 or T6 under the orthogonal condition; (b) is for the leftward movements from S1 to T5 under the parallel condition; (c) is for the rightward movements from S2 to T7 or T8 under the orthogonal condition; (1) and (2) are for the subjects T.S. and K.T., respectively. In Fig. 5, 10-trial measured data overlap each other. From Fig. 5, the following results were obtained: (1) the hand joint was nearly extended $\left(\theta_{3}=0\right)$ at the start position, whereas it was flexed to the desired angle at the final position; (2) the above result was independent of the start and
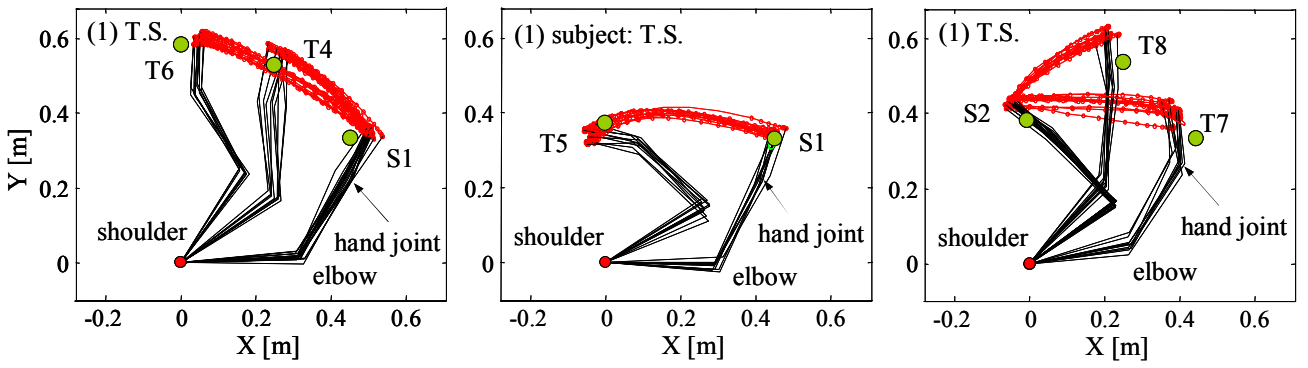

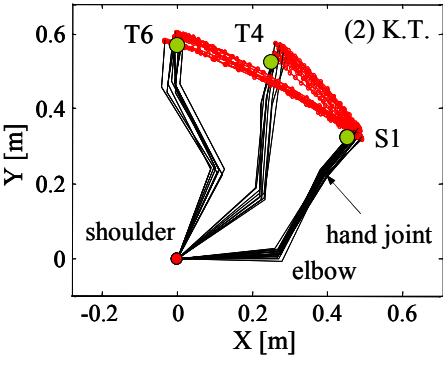

(a) Paths [S1-T4 \& T6:orthogonal]

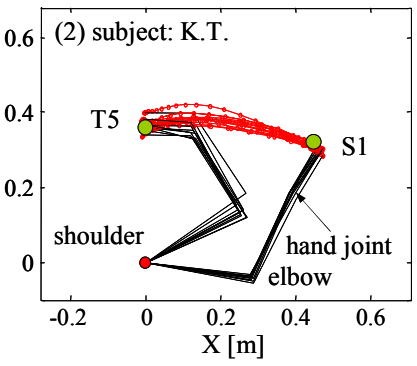

(b) Paths [S1-T5:parallel]

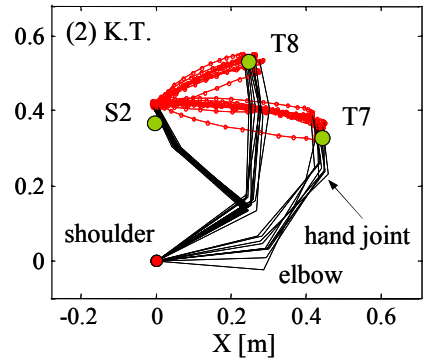

(c) Paths [S2-T7 \& T8:orthogonal]

Fig. 5 Hand paths derived from the constrained reaching movements measured experimentally. 
target positions and the subjects. On the other hand, we already clarified that for unconstrained reaching movements, the human hand-joint angle hardly changes over the entire movement regardless of start and target positions and subjects ${ }^{(8)}$. Therefore, the above measured results give us the following implications: (1) for unconstrained reaching movements, the human hand-joint angle is kept almost constant over the entire movement because of the freezing-like mechanism; (2) for constrained reaching movements, the human hand-joint angle can be changed to the desired value during the movement, even though the freezing-like mechanism functions so as to prevent the hand-joint angle from changing. Therefore, if the proposed three-joint model can be a general and plausible model for the human three-joint arm's control mechanism, it should be able to reproduce the implications mentioned above. In Fig. 5, the start and final positions of the measured hand paths did not always correspond to the actual start and target positions (green circles). These results can be thought to be caused primarily by the subjects, not the two-link mechanical manipulator in the experimental apparatus. That is because in measurements of other reaching movements using the same experimental apparatus, the start and final positions of the measured hand paths corresponded well to the actual start and target positions ${ }^{(8)}$.

\subsection{Trajectory reproduction for constrained reaching movements}

Figure 6 shows the optimal hand paths predicted by minimizing the energy term $W$ in the criterion function Eq. (4) as a parameter of each of three kinds of weights $\left(\omega_{1}, \omega_{2}, \omega_{3}\right)$; (a) represents the optimal hand paths predicted by minimizing the moment power of joints as a parameter of $\omega_{1}\left(\omega_{2}=\omega_{3}=0\right)$ for the leftward movements from S1 to T4 under the orthogonal condition; (b) represents those predicted by minimizing the energy for sustaining the torque of joints as a parameter of $\omega_{2}\left(\omega_{1}=\omega_{3}=0\right)$ for the leftward movements from S1 to T5 under the parallel condition; (c) represents those predicted by minimizing the energy consumed by viscous resistance as a parameter of $\omega_{3}\left(\omega_{1}=\omega_{2}=0\right)$ for the rightward movements from S2 to T7 under the orthogonal condition; (1) and (2) are for the subjects T.S. and K.T., respectively. For comparison, the experimentally measured hand paths (black lines) are also added to Fig. 6. The optimization was executed by substituting the values of the state variables $\boldsymbol{x}_{0}$ and $\boldsymbol{x}_{t f}$ into the boundary conditions of Eq. (9) and solving the twopoint boundary-value problem of Eq. (8) based on a Newton-like method. The first three
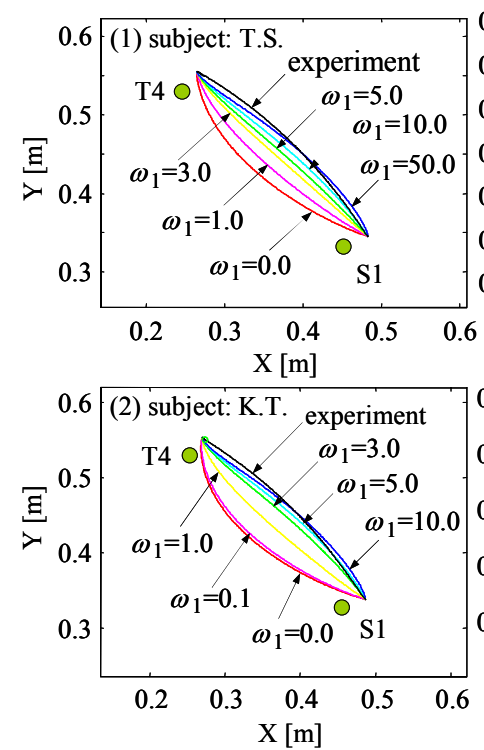

(a) Joint moment power (S1-T4: orthogonal)

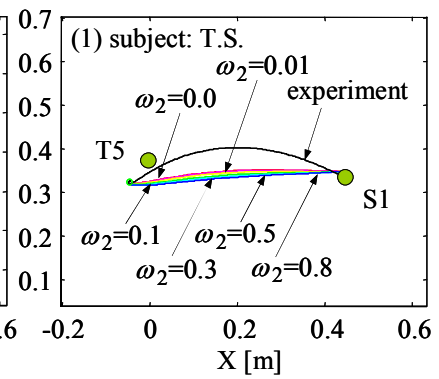

0.6 (2) subject: K.T.

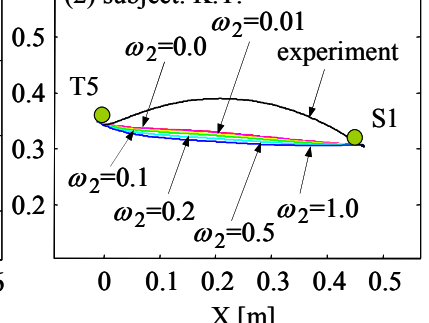

(b) Torque-sustaining energy (S1-T5: parallel)
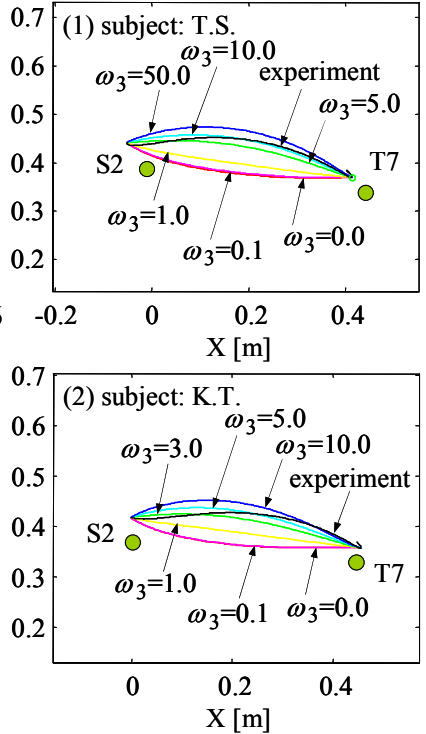

(c) Energy consumed by viscous resistance (S2-T7: orthogonal)

Fig.6 Influence of the energy weights on the hand paths predicted by minimizing the energy term $W$. 
components $\left(\theta_{1}, \theta_{2}, \theta_{3}\right)$ of the state variables $\boldsymbol{x}_{0}$ and $\boldsymbol{x}_{t f}$ were derived from one of the measured paths, and the other six components were fixed to zero as the arm was in a state of rest at the start and final positions. The movement durations for Fig. 6 (a), (b), and (c) were $t_{f}=781,1019,807 \mathrm{msec}$, respectively, for subject T.S. and $t_{f}=767,756,787 \mathrm{msec}$, respectively, for subject K.T.; the values of the weight matrices $\boldsymbol{Q}$ and $\boldsymbol{R}$ were fixed to $Q_{11} \sim Q_{99}=10^{7}$ and $R_{11}=R_{22}=R_{33}=1.0$, and the weight value $\omega_{4}$ was fixed to 0.01 . Since the weight $\omega_{4}$ of the torque-change term in the criterion function was set to a much smaller value than the other weights $\left(\omega_{1}, \omega_{2}, \omega_{3}\right)$, minimizing the criterion function could be practically regarded as minimizing only the energy term. This calculation was executed to verify which energy minimization makes it possible to reproduce the constrained reaching movements. From Fig. 6 the following results were obtained; (1) as for minimizing the moment power of joints (Fig.6 (a)), the reproduced hand path changed gradually from a concave curve to a convex one with an increase in $\omega_{1}$ and agreed nearly with the measured path at a certain value of $\omega_{l}$; (2) as for minimizing the energy for sustaining the torque of joints (Fig.6 (b)), the reproduced hand path maintained almost the same concave curve regardless of the value of $\omega_{2}$ and never agreed with the measured path; (3) as for minimizing the energy consumed by viscous resistance (Fig.6 (c)), the reproduced hand path changed gradually from a concave curve to a convex one with an increase in $\omega_{3}$ and agreed nearly with the measured path at a certain value of $\omega_{3}$; (4) the above results were independent of the subjects. These results make us expect a possibility that, if the value of $\omega_{1}$ or $\omega_{3}$ is appropriately selected once for each subject, the minimization of the moment power of joints or the energy consumed by viscous resistance will make it possible to reproduce the constrained reaching movements measured experimentally regardless of the start and target positions. In Fig. 6, the start and final positions of the reproduced hand paths corresponded exactly to those of the measured hand paths, whereas they did not always correspond to the actual start and target positions (green circles). The former is because the reproduced hand paths were calculated by using the state variables $\boldsymbol{x}_{0}$ and $\boldsymbol{x}_{t f}$ derived from one of the measured paths, and the latter is because the start and final positions of the measured hand paths themselves did not always correspond to the actual start and target positions as mentioned above.

Figure 7 shows the comparison between the hand paths (dotted lines) derived from the constrained reaching movements measured experimentally and the hand paths (solid lines)
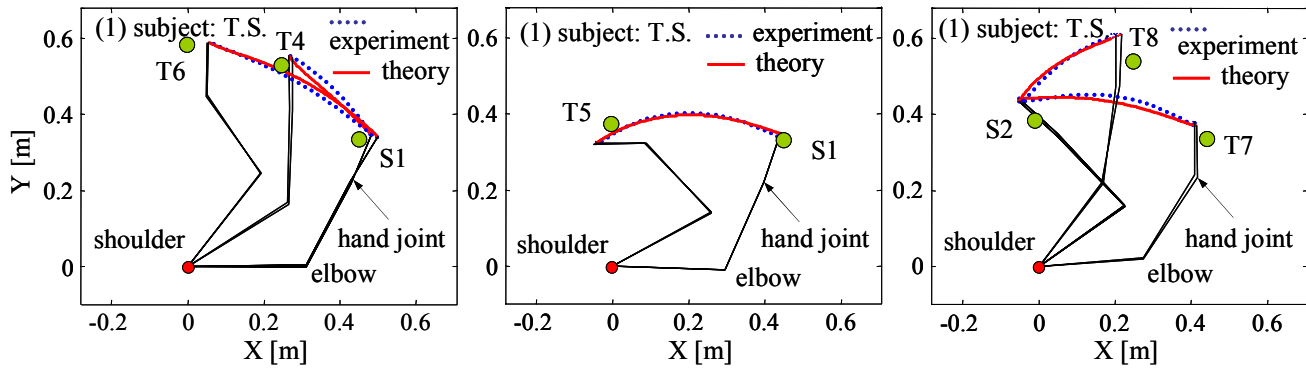

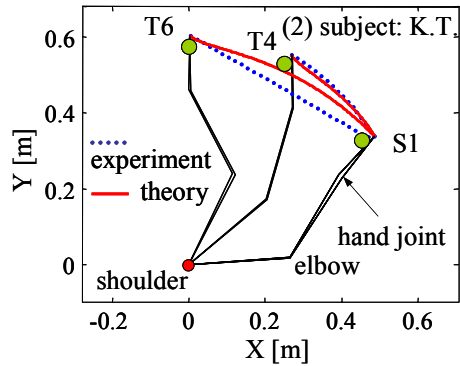

(a) Paths [S1-T4 \& T6: orthogonal]

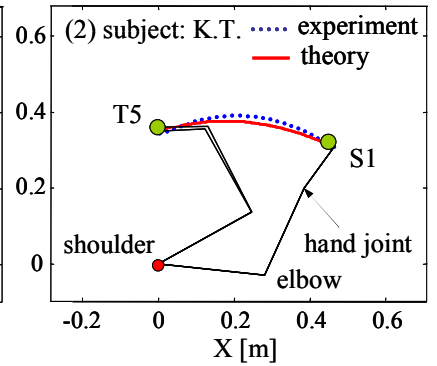

(b) Paths [S1-T5: parallel]

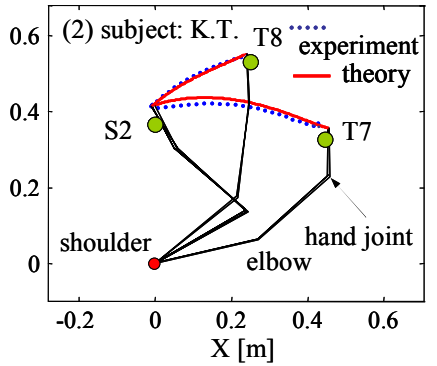

(c) Paths [S2-T7 \& T8: orthogonal]

Fig.7 Comparison between the measured hand paths and the theoretical ones predicted by minimizing the moment power of joints at a constant value of $\omega_{1}$ 
predicted by minimizing the moment power of joints $\left(\omega_{2}=\omega_{3}=0\right)$ at a constant value of $\omega_{1}$; (a) is for the leftward movements from S1 to T4 or T6 under the orthogonal condition; (b) is for the leftward movements from S1 to T5 under the parallel condition; (c) is for the rightward movements from S2 to T7 or T8 under the orthogonal condition; (1) and (2) are for the subjects T.S. and K.T., respectively. The value of $\omega_{1}$ was 3.0 for subject T.S. and 5.0 for subject K.T.; the movement durations were $t_{f}=781,1019,940,807,851 \mathrm{msec}$ for T4, T5, T6, T7, and T8, respectively, for subject T.S. and $t_{f}=767,756,845,787,663 \mathrm{msec}$ for subject K.T.; the values of the weight matrices $\boldsymbol{Q}$ and $\boldsymbol{R}$ were fixed to $Q_{11} \sim Q_{99}=10^{7}$ and $R_{11}=$ $R_{22}=R_{33}=1.0$; the weight value $\omega_{4}$ was fixed to 0.01 . This calculation was executed to verify how the hand paths predicted at the constant value of $\omega_{1}$ agree with those measured experimentally regardless of the start and target positions. From Fig. 7, the following results were obtained: (1) the predicted hand paths agreed well with the measured ones regardless of the start and target positions, except that for the leftward movement from S1 to T6 (Fig. 7 (a)(2)), the predicted hand path deviated somewhat from the measured one; (2) the predicted hand postures at the final points agreed well with the measured ones regardless of the target positions and the desired hand postures. Moreover, we also examined how the hand paths and the hand postures predicted by the minimization of the energy consumed by viscous resistance $\left(\omega_{1}=\omega_{2}=0\right)$ at the same value of $\omega_{3}$ as the value of $\omega_{1}$ agree with those measured experimentally, and confirmed that the derived results are the same as the above two results. Therefore, the result (1) means that, if the minimization of the moment power of joints or that of the energy consumed by viscous resistance is executed, the proposed model can reproduce measured hand paths for most of the constrained reaching movements by using the constant weight value of $\omega_{1}$ or $\omega_{3}$. The result (2) means that the proposed model can move its hand joint during the movement in the same way as the actual human arm system, even though the feedback mechanism in the model's hand joint functions so as to prevent its hand-joint angle from changing. The hand path deviation in the result (1) can be thought to be caused by using the constant weight value of $\omega_{1}$ to make the model more general and plausible. A possible way to reduce the deviation would be to optimize the weighted sum of the three kinds of energy costs and to search three kinds of constant weight values of $\omega_{1}, \omega_{2}$, and $\omega_{3}$, though it is a troublesome task to find the constant weight values. Figs. 6 and 7 resulted from optimizing one of the three kinds of energy costs. In addition, we also confirmed that the proposed model never reproduces the measured hand paths without its hand-joint's feedback controller $(k a=0.0)$ because its hand joint is moved more than necessary under this condition, and that the excessive movement of the model's hand joint is never improved by changing the model's weight values $\left(\omega_{1}, \omega_{2}, \omega_{3}, \omega_{4}\right.$, and $\left.\boldsymbol{R}\right)$. This implies that the hand-joint's feedback mechanism is indispensable for reproducing human constrained reaching movements as well as human unconstrained reaching movements.

Figure 8 shows the comparison between the joint angle profiles (dotted lines) derived from the constrained reaching movements measured experimentally and the joint angle profiles (solid lines) predicted by minimizing the energy consumed by viscous resistance $\left(\omega_{1}=\omega_{2}=0\right)$; (a) and (d) are for the leftward movements from S1 to T6 and T4, respectively, under the orthogonal condition; (b) and (e) are for the leftward movements from S1 to T5 under the parallel condition; (c) and (f) are for the rightward movements from S2 to T7 and T8, respectively, under the orthogonal condition; (1) and (2) are for the subjects T.S. and K.T., respectively. The value of $\omega_{3}$ was fixed to 3.0 for subject T.S. and 5.0 for subject K.T.; the movement durations were $t_{f}=940,1019,807 \mathrm{msec}$ for the targets T6, T5, and T7, respectively, for subject T.S. and $t_{f}=767,756,663 \mathrm{msec}$ for the targets T4, T5, and T8, respectively, for subject K.T.; the values of the weight matrices $\boldsymbol{Q}$ and $\boldsymbol{R}$ were fixed to $Q_{11} \sim$ $Q_{99}=10^{7}$ and $R_{11}=R_{22}=R_{33}=1.0$; the weight value $\omega_{4}$ was fixed to 0.01 . This calculation was executed to verify how the predicted hand-joint angle profiles, i.e., the predicted angular transition characteristics for the hand joint over the entire movement, agree with those 
measured experimentally regardless of the start and target positions. From Fig. 8, the following results were obtained: (1) the predicted shoulder- and elbow-joint angle profiles agreed well with the measured ones regardless of the start and target positions, except that for the leftward movement from S1 to T5 (Fig. 8 (e)), the predicted elbow-joint angle profile deviated somewhat from the measured one; (2) the predicted hand-joint angle profiles agreed well with the measured ones regardless of the start and target positions, except that for the rightward movement from S2 to T7 (Fig. 8 (c)), the predicted hand-joint angle profile deviated somewhat from the measured one. Moreover, we also examined how the joint angle profiles predicted by the minimization of the moment power of joints $\left(\omega_{2}=\omega_{3}=0\right)$ at the same value of $\omega_{1}$ as in Fig. 7 agree with those measured experimentally, and confirmed that the derived results are the same as the above two results. Therefore, the above results mean that by using the constant weight value of $\omega_{1}$ or $\omega_{3}$, the proposed model can reproduce the measured angular transition characteristics for the hand joint as well as the measured hand paths for most of the constrained reaching movements. The joint angle deviations in the above results can be thought to be caused by two factors: one is to use the constant weight value of $\omega_{3}$ to make the model more general and plausible; the other is to use the scalar weight $\omega_{3}$ to make the model as simple as possible. If a diagonal weight matrix like $\boldsymbol{Q}$ and $\boldsymbol{R}$ is substituted for the scalar weight $\omega_{3}$, it can provide the three joints with their individually different weight values; then, the different weight values would make it possible to reduce these joint angle deviations, though the model becomes more complicated.

Therefore, the above results (Figs. 6, 7, and 8) suggest that the proposed model has the ability to reproduce human three-joint arm's constrained reaching movements accompanied by evident motion in the hand joint as well as unconstrained ones accompanied by little motion: in other words, it can be a general and plausible model for the human three-joint arm's control mechanism.

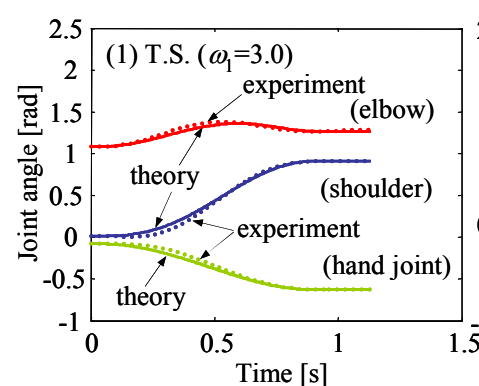

(a) leftward [S1-T6: orthogonal]

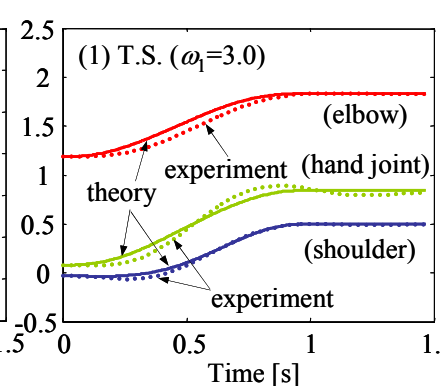

(b) leftward [S1-T5: parallel]

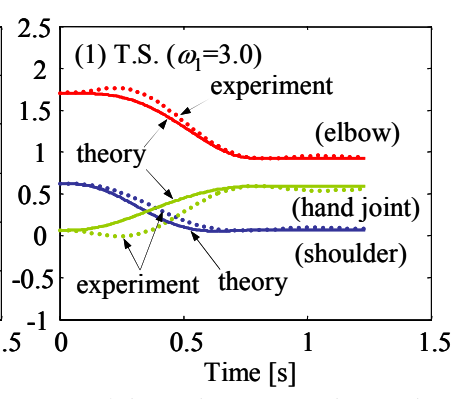

(c) rightward [S2-T7: orthogonal]

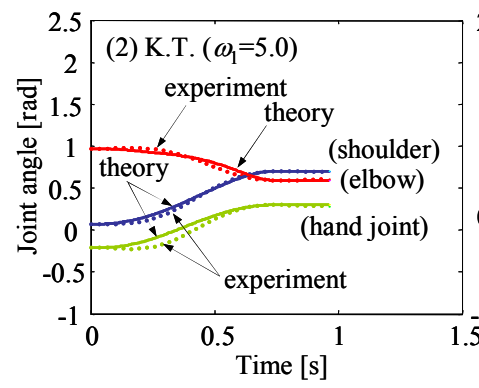

(d) leftward [S1-T4: orthogonal]

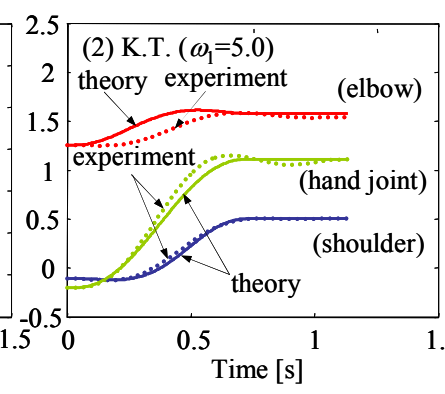

(e) leftward [S1-T5: parallel]

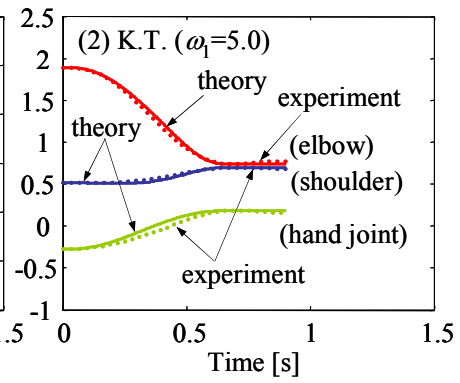

(f) rightward [S2-T8: orthogonal]

Fig.8 Comparison between the measured joint angle profiles and the theoretical ones predicted by minimizing the energy consumed by viscous resistance at a constant value of $\omega_{3}$

\section{Discussion}

Figures 6,7 , and 8 clarified that the proposed model was able to reproduce the human 
three-joint arm's constrained reaching movement characteristics. In this chapter, in terms of the forces acting on the hand joint, we consider computationally how the hand-joint's feedback mechanism functions to reproduce those characteristics.

The forces acting on the model's hand joint consist of three kinds of torques: hand-joint torque $\tau_{3}$, feedback torque $\tau_{3}{ }^{*}$, and hand-joint moment (hereafter called inertial torque) produced by the inertial force acting on the model's hand when the model's arm moves at an accelerated or decelerated rate. Figures 9 and 10 show the profiles of the three kinds of torques predicted by minimizing the moment power of joints $\left(\omega_{2}=\omega_{3}=0\right)$ at a constant weight value of $\omega_{1}$ for the unconstrained and constrained reaching movements, respectively; the blue, red, and green lines represent the hand-joint torque $\tau_{3}$, the feedback torque $\tau_{3}{ }^{*}$, and the inertial torque, respectively; the black lines represent the sum of the three kinds of torques, i.e., resultant torque. This calculation was executed to elucidate the difference in the torque profile between the unconstrained and constrained reaching movements with the same start and target positions. The three kinds of torques for the unconstrained reaching movements were derived from the calculation results in the previous work ${ }^{(8)}$. In Figs. 9 and 10, (a) and (d) are for the leftward movements from S1 to T6 and T4, respectively, and the constrained movements in Fig. 10 are under the orthogonal condition; (b) and (e) are for the leftward movements from S1 to T5, and the constrained ones are under the parallel condition; (c) and (f) are for the rightward movements from S2 to T7 and T8, respectively, and the constrained ones are under the orthogonal condition; (1) and (2) are for the subjects T.S. and K.T., respectively. The value of $\omega_{1}$ was fixed to 3.0 for subject T.S. and 5.0 for subject K.T.; the movement durations were $t_{f}=940,1019,807 \mathrm{msec}$ for T6, T5, and T7, respectively, for subject T.S. and $t_{f}=767,756,663 \mathrm{msec}$ for T4, T5, and T8, respectively, for subject K.T.; the values of the weight matrices $\boldsymbol{Q}$ and $\boldsymbol{R}$ were fixed to $Q_{11} \sim Q_{99}=10^{7}$ and $R_{11}=$ $R_{22}=R_{33}=1.0$; the weight value $\omega_{4}$ was fixed to 0.01 .

From Fig. 9 (unconstrained reaching movements), the following results were obtained: (1) the hand-joint torque $\tau_{3}$ acted in the direction opposite to the inertial torque; (2) the feedback torque $\tau_{3}{ }^{*}$ tended to act in the direction in which the imbalance between the handjoint torque and the inertial torque was corrected; (3) the sum of the three kinds of torques averaged nearly zero over the entire movement. The results (1) and (2) mean that for the

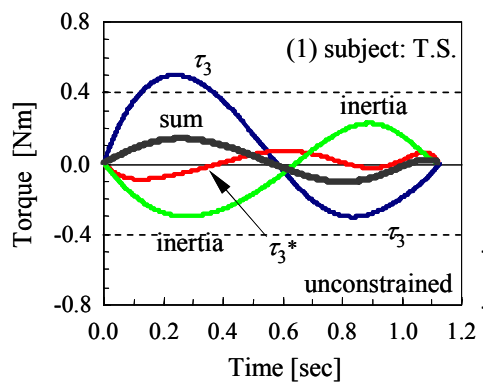

(a) leftward [S1-T6]

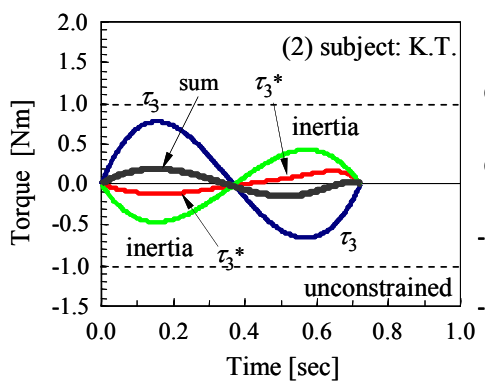

(d) leftward [S1-T4]

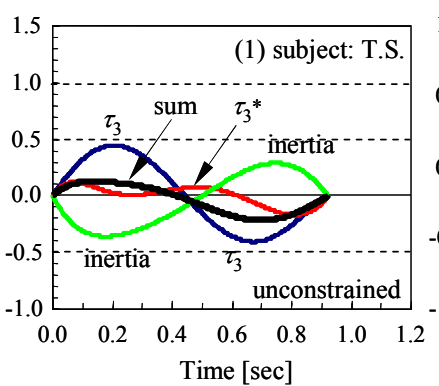

(b) leftward [S1-T5]

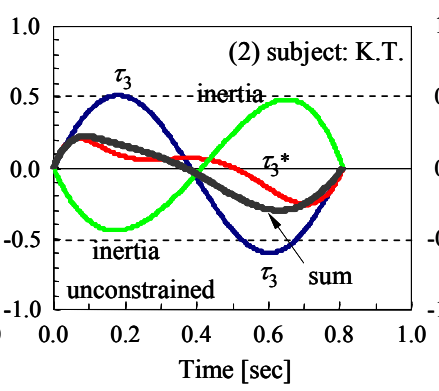

(e) leftward [S1-T5]

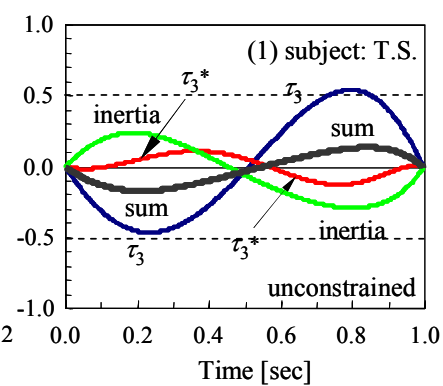

(c) rightward [S2-T7]

Fig.9 Relation between the hand-joint, feedback, and inertial torques predicted by minimizing the moment power of joints at a constant weight value of $\omega_{I}$ (unconstrained movements) 


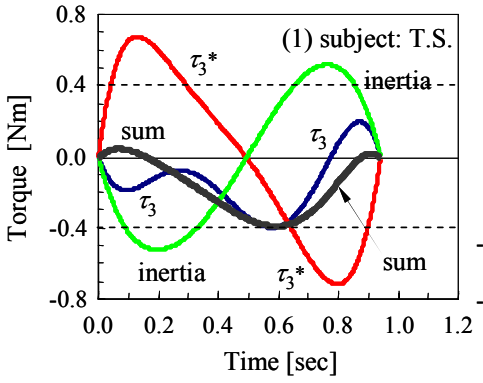

(a) leftward [S1-T6: orthogonal]

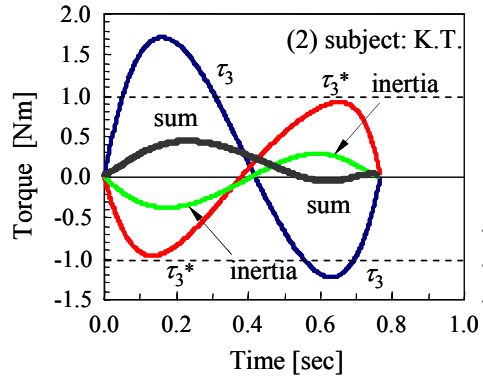

(d) leftward [S1-T4: orthogonal]

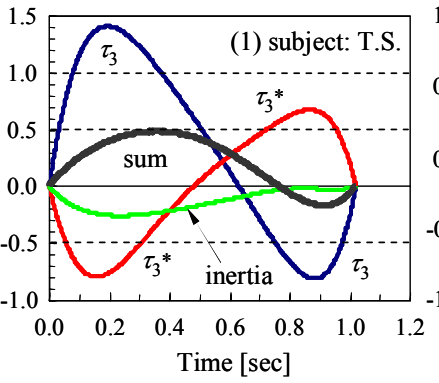

(b) leftward [S1-T5: parallel]

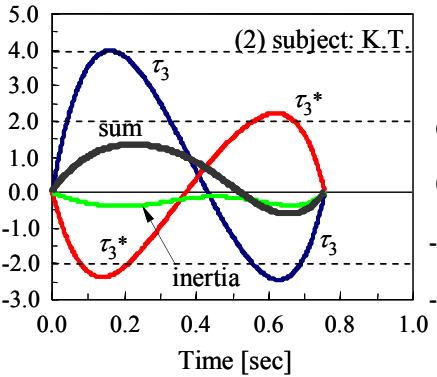

(e) leftward [S1-T5: parallel]

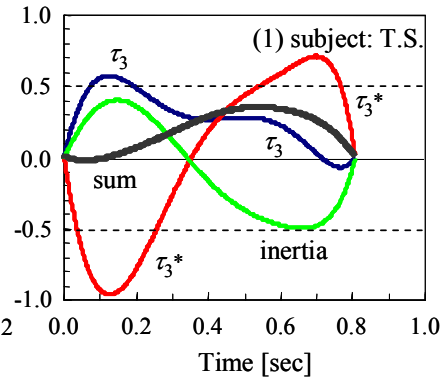

(c) rightward [S2-T7: orthogonal]

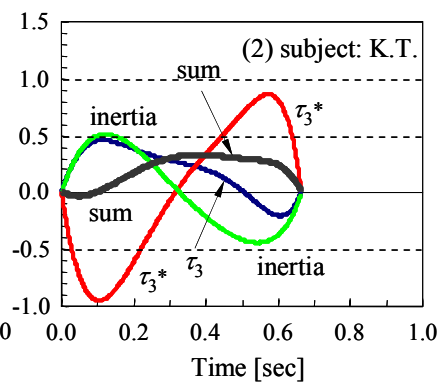

(f) rightward [S2-T8: orthogonal]

Fig.10 Relation between the hand-joint, feedback, and inertial torques predicted by minimizing the moment power of joints at a constant weight value of $\omega_{1}$ (constrained movements)

unconstrained reaching movements, as soon as the model's hand joint is about to move because of the imbalance between the hand-joint torque and the inertial torque, the handjoint's feedback mechanism functions so as to produce the feedback torque in the direction opposite to the hand-joint motion. That will be why the model's hand-joint angle hardly changes over the entire movement. Therefore, the direction of the feedback torque depends on the imbalance between the hand-joint torque and the inertial torque. The result (3) means that for the unconstrained reaching movements, the resultant torque around the hand joint averages nearly zero over the entire movement; this will be the direct cause that makes the model's hand joint hardly move. We already clarified that the proposed model never reproduces unconstrained reaching movement characteristics measured experimentally without its hand-joint's feedback controller $(k a=0.0)^{(8)}$. That will be because only the two kinds of torques, the hand-joint and inertial torques, are not enough to control the model's hand joint so as to prevent it from moving. In other words, the optimal relation among the three kinds of torques, which is determined by the proposed model, will be required to prevent the model's hand joint from moving.

From Fig. 10 (constrained reaching movements), the following results were obtained: (1) the hand-joint torque $\tau_{3}$ did not always act in the direction opposite to the inertial torque, in other words, the relationship between the two kinds of torques was more complicated than for the unconstrained reaching movements; (2) the feedback torque $\tau_{3}{ }^{*}$ tended to act in the direction in which the imbalance between the hand-joint torque and the inertial torque was corrected; (3) the sum of the three kinds of torques averaged not zero but a biased value over the entire movement. The results (1) and (2) mean that the hand-joint's feedback mechanism basically tends to produce the feedback torque in the direction opposite to the hand-joint motion caused by the imbalance between the hand-joint torque and the inertial torque. The complexity of the relationship between the hand-joint torque and the inertial torque will be because the model has to bend its hand joint to the desired angle in spite of the existence of the hand-joint's feedback mechanism. The result (3) means that for the constrained reaching movements, the resultant torque around the hand joint averages a biased value over the entire movement; this will be the direct cause that makes it possible 
for the model's hand to take the desired posture in spite of the existence of the hand-joint's feedback mechanism. We also clarified that the proposed model never reproduces the constrained reaching movement characteristics measured experimentally without its handjoint's feedback controller $(k a=0.0)$. That will be because only the two kinds of torques, the hand-joint and inertial torques, are not enough to control the model's hand joint so as to move it to the desired angle. In other words, the optimal relation among the three kinds of torques, which is determined by the proposed model, will be required to move the model's hand joint to the desired angle.

Therefore, from the computational point of view, it can be concluded that the difference in the hand-joint's angular transition characteristic between the unconstrained and constrained reaching movements is caused by the average of the resultant torque around the hand joint over the entire movement. Judging from the results that the proposed model can reproduce human constrained reaching movement characteristics (Figs. 6, 7, and 8), there can also be a possibility that the three kinds of torques will be involved in human hand-joint's movement characteristics regardless of the type of reaching movements, unconstrained or constrained ones. However, the detail verification will be our future work.

\section{Conclusion}

In order to validate the proposed three-joint arm's optimal control model with its handjoint's feedback mechanism, this paper applied it to reproducing human constrained reaching movements in which the hand had to take a specified posture at the target point. The following results were obtained: (1) the proposed model was able to move its hand joint regardless of the start and target positions and the hand postures at the final points in spite of the existence of its feedback mechanism that prevents its hand-joint angle from changing; (2) the hand trajectories and the hand-joint's angular transition characteristics predicted by the model agreed well with those measured experimentally for most of the constrained reaching movements. These results suggest that the proposed model has the ability to simulate human three-joint arm's reaching movements regardless of whether they are unconstrained or constrained, i.e., it can be effective as a general and plausible model for the human three-joint arm's control mechanism. Moreover, we considered computationally that the hand-joint's angular transition characteristic depends on the average of the resultant torque around the hand joint over the entire movement.

\section{References}

(1) Flash, T. and Hogan, N., The Coordination of Arm Movements: An Experimentally Confirmed Mathematical Model, J. Neuroscience, Vol. 5 (1985), pp. 1688-1703.

(2) Uno, Y., Kawato, M., and Suzuki, R., Formation and Control of Optimal Trajectory in Human Multijoint Arm Movement - Minimum Torque-Change Model, Biol. Cybern., Vol. 61 (1989), pp. 89-101.

(3) Nakano, E., Imamizu, H., Osu, R., Uno, Y., Gomi, H., Yoshioka, T., and Kawato, M., Quantitative Examination of Internal Representations for Arm Trajectory Planning: Minimum Commanded Torque Change Model, J. Neurophysiol., Vol. 81 (1999), pp. 2140-2155.

(4) Kashima, T. and Isurugi, Y., Trajectory Formation in Human Arm Movement, Transaction of SICE, Vol. 31, No. 9 (1995), pp. 1416-1422.

(5) Kashima, T. and Isurugi, Y., Human Arm Trajectory Formation, Transaction of SICE, Vol. 34, No. 10 (1998), pp. 1440-1447.

(6) Harris, C.M. and Wolpert, D.M., Signal- Dependent Noise Determines Motor Planning, Nature, Vol. 394, No. 20 (1998), pp. 780-784.

(7) Matsui, T., Honda, M., and Nakazawa, N., A New Optimal Control Model for Reproducing Human Arm's Two-Point Reaching Movements: A Modified Minimum Torque Change 
Model, Proceedings of the 2006 IEEE International Conference on Robotics and Biomimetics (2006), pp.1541-1546.

(8) Matsui, T., Takeshita, K., and Shibusawa, T., An Optimal Control Model for Human Three-Joint Arm's Reaching Movements Including Hand Joint's Freezing Mechanism, Transactions of the Japan Society of Mechanical Engineers, C, Vol. 76, No. 762 (2010), pp. 313-322.

(9) Bernstein, N., The Co-ordination and Regulation of Movements, Pergamon Press, London (1967).

(10) Gatev, U., Role of Inhibition in the Development of Motor Co-ordination in Early Childhood, Developmental Medicine and Child Neurology, Vol. 14 (1972), pp. 336-341.

(11) Nielsen, J. and Kagamihara, Y., The Regulation of Disynaptic Reciprocal Ia Inhibition during Co-contraction of Antagonistic Muscles in Man, J. Physiology, Vol. 456 (1992), pp. 373-391. 